\title{
THE VALUE OF CAROTID INTIMA-MEDIA MEASUREMENTS DURING THE PERIODS OF ATTACK AND REMISSION OF MIGRAINE DISEASE AND TENSION-TYPE HEADACHE IN DIFFERENTIAL DIAGNOSIS
}

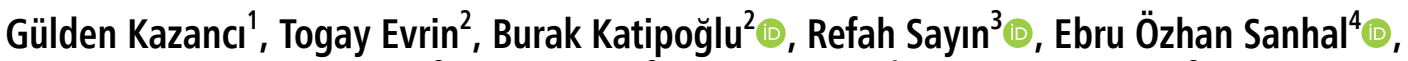 \\ Ertuğrul Kazanci ${ }^{2}$, Fatih Demir ${ }^{2}$, Semih Korkut ${ }^{4} \odot$, Lukasz Szarpak ${ }^{6} \odot$ \\ 'Department of Emergency Medicine, University of Health Sciences, Ankara Training and Research Hospital, Ankara, Turkey \\ ${ }^{2}$ Department of Emergency Medicine, Ufuk University, Faculty of Medicine, Ankara, Turkey \\ ${ }^{3}$ Department of Neurology, Ufuk University, Faculty of Medicine, Ankara, Turkey \\ ${ }^{4}$ Department of Radiology, Ufuk University, Faculty of Medicine, Ankara, Turkey \\ ${ }^{5}$ Department of Emergency Medicine, University of Health Sciences, İstanbul Kartal Dr Lütfi Kırdar Training and Research Hospital, İstanbul, Turkey \\ ${ }^{6}$ Faculty of Medicine, Lazarski University, Warsaw, Poland
}

\begin{abstract}
INTRODUCTION: In Turkey, 2\% of emergency department admissions are migraine and there is no specific biomarker with differential diagnostic value in patients presenting with headache. There are studies showing that the blood-brain barrier deteriorates during migraine attacks, the risk of atherosclerosis is increased in migraine patients and it is associated with cardiovascular and cerebrovascular diseases. In light of this information, the value of carotid intima-media thickness (CIMT) measurements in the differential diagnosis of tension and migraine headache was investigated.
\end{abstract}

MATERIAL AND METHODS: The study group consisting of 20 tension headache, 23 migraine attacks, 20 remission migraine patients admitted to the emergency department and neurology outpatient clinic of Ridvan Ege Hospital between February and September 2017 and control group of 21 healthy volunteers were compared. Carotid intima-media thickness (CIMT) of all participants was measured by specialist radiologist. The data obtained were analysed using SPSS 15.0 statistical program.

RESULTS: There was no significant difference between the participants in terms of age and gender. When the carotid intima-media thickness was analysed between the four groups and according to the presence and type of migraine, no significant difference was found $(p=0.489)$.

CONCLUSIONS: This is the first study in the literature which evaluates the relationship between subclinical atherosclerosis and tension-type headache and migraine by examining patients with tension-type headache and migraine disease in both episode and remission period. According to the results obtained, migraine and tension headache is not associated with subclinical atherosclerosis. However, larger-scale studies are required in this sense.

KEY WORDS: migraine; tension-type headache; carotid intima-media thickness; emergency medicine

Disaster Emerg Med J 2019; 4(3): 109-115 


\section{INTRODUCTION}

Headache is the most common painful condition affecting people. It is also a symptom that can accompany systemic diseases as well as neurological diseases. Headache is one of the most common symptoms in the community. The frequency of headache in patients admitted to the emergency department is approximately 3-5\%. Among these, about $20-55 \%$ of patients have a primary headache, and approximately $40-50 \%$ of patients have systemic diseases. It has been found that about $2 \%$ of patients who admitted to the emergency department with headache in Turkey was diagnosed with migraine [1]. In the prevalence studies conducted in Turkey, the prevalence of migraine was $16.4 \%$ and the frequency of tension headache was $31.7 \%$ [2]. Approximately $3.8 \%$ of patients presenting to the emergency department with headache have an underlying cause and have been diagnosed with secondary headache [3]. Therefore, it is very important to make a primary/secondary distinction. In the International Headache Society, IHS classification system, a primary headache is classified into 4 groups, which are migraine, tension headache, paroxysmal hemicrania and other primary headaches.

Migraine, one of the primary headaches, is a very common disease in the community with severe headache attacks. It is more common in women and it affects about $12 \%$ of the population. It is known that approximately $20 \%$ of women and $8 \%$ of men have migraine [4]. The most common type of primary headache is tension-type headache. In Turkey, its annual prevalence is about 32\% [4]. Although tension-type headache is more common than migraine, it causes a relatively mild headache and is more in the background at presentation to the physician. The full pathophysiology of tension-type headache has not been explained yet but psychological stress is the most common underlying cause.

In recent studies, migraine has been shown to be associated with comorbid vascular risk factors like hypertension, obesity, hyperlipidaemia, insulin resistance which are also associated with atherosclerosis. Atherosclerosis and associated diseases are still the most important causes of morbidity and mortality worldwide [5]. The most important finding in the early stage of atherosclerotic disease is increased intima-media thickness in the artery wall [6]. In recent years, studies on carotid artery intima-media thickness (CIMT) revealed that it has been reported to be an indicator of subclinical ath- erosclerosis and correlated with myocardial infarction, cerebrovascular diseases and peripheral artery diseases $[7,8]$. In a study conducted in paediatric migraine patients, carotid intima-media thickness measurements were shown to be higher in patients with migraine than in the control group [9]. In another study conducted with 25 adult female migraine patients, carotid intima-media thickness was higher compared to the control group and it was concluded that migraine patients were prone to subclinical atherosclerosis [10].

Based on all these observations, the aim of this study was to evaluate the value of carotid intima-media thickness in the differential diagnosis of migraine patients presenting to the emergency department and neurology outpatient clinic in the episodes of attack and remission and the patients with tension-type headache, and to evaluate the risk of subclinical atherosclerosis in these patients.

\section{MATERIALS AND METHODS}

The study group was selected from patients aged between 18-65 years who applied to the emergency department and neurology outpatient clinic of Faculty of Medicine, Ufuk University Dr Ridvan Ege Training and Research Hospital with a headache. The participants were recruited from February 2017 to September 2017. All patients admitted for headache were evaluated according to the international headache classification, the latest beta version of the International Headache Society, following neurological and other systemic examinations. Secondary headaches were ruled out and migraine attacks, tension-type headaches and migraine in remission subgroups were formed.

Patients with a history of malignancy, hypertension, cardiovascular disease, cerebrovascular disease, diabetes mellitus, chronic renal failure, liver failure, active infection, haematologic disease, pregnancy, pulmonary embolism, smoking/alcohol addiction and autoimmune disease were not included in this study. Signed consent forms were obtained from all participants and all participants were informed about the study.

Demographic information, medical history, family history of migraine, type and character of headache, duration and number of migraine attacks, duration of migraine disease, VAS (Visual Analogue Scale) score, MIDAS (Migraine Disability Rating Scale) score and carotid intima-media thick- 
ness were recorded. Questionnaires were organized and recorded for each patient and control group. Intima-media thicknesses of both carotid arteries were recorded by the ultrasound guided by the radiologist without the knowledge of which group the participants were from.

20 patients with tension headache, 23 with migraine attacks and 20 remission migraine patients were included in the study. The control group according to the patients in the study group was composed of a total of 21 healthy volunteers. In total, 84 participants were included in the study (Tab. 1).

This study was conducted with the approval of Ufuk University Faculty of Medicine Ethics Committee for Non-Drug and Non-Medical Device Research.

Among the study groups, 9 men (45\%) and 11 women (55\%) were in the tension-type headache (TTH) group, 4 men (20\%) and 16 women $(80 \%)$ were in the migraine remission period group, and 6 men (26.08\%) and 17 women (73.91\%) were in the migraine attack group. The volunteers in the control group were 11 males (53\%) and 10 females $(47 \%)$.

The total number of participants with remission and attack periods of migraine was 43 . Of the 84 participants, 41 had no migraine. Twenty of these 41 participants consisted of tension-type attack group and 21 of them were a control group. Those with a diagnosis of migraine constitute $51.2 \%$ of the whole study group and $48.8 \%$ without a diagnosis of migraine (Tab. 2).

Of the 43 participants with migraine, 22 (51.2\%) had migraine with aura and 21 (48.8) had migraine without aura (Tab. 3).

Carotid intima-media thickness (CIMT) measurements were performed with General Electric Logiq 7 ultrasound device using a $5-12 \mathrm{MHz}$ superficial probe. For carotid artery imaging, the patient was placed in the supine position and the angle was approximately $20^{\circ}$ to the opposite side of the neck. Measurements were obtained in the longitudinal plane from the distance defined as between vascular lumen echogenicity and media/adventitia echogenicity by B-mode examination. CIMT measurements were performed in the distal $2 \mathrm{~cm}$ segment of the main carotid arteries prior to the bulbous, approximately $1 \mathrm{~cm}$ in length and only by evaluating the posterior (distal) wall. The mean CIMT was obtained by calculating the mean of three measurements from both carotid arteries.

Table 1. Distribution of participants between
groups
\begin{tabular}{|l|c|c|}
\hline & Number & Percentile \\
\hline Tension type headache & 20 & $\% 23.8$ \\
\hline Migraine in remission & 20 & $\% 23.8$ \\
\hline Migraine attack & 23 & $\% 27.4$ \\
\hline Control group & 21 & $\% 25$ \\
\hline Total & $\mathrm{n}=84$ & $\% 100$ \\
\hline
\end{tabular}

Table 2. Distribution of migraine disease among participants

\begin{tabular}{|l|c|c|}
\hline & Number & Percentile \\
\hline Migraine & 43 & $\% 51.2$ \\
\hline No migraine & 41 & $\% 48.8$ \\
\hline
\end{tabular}

Table 3. Distribution by migraine-type
\begin{tabular}{|l|c|c|}
\hline \multicolumn{1}{|c|}{ Migraine type } & Number & Percentile \\
\hline With aura & 22 & $\% 51.2$ \\
\hline Without aura & 21 & $\% 48.8$ \\
\hline Total & 43 & $\% 100$ \\
\hline
\end{tabular}

\section{STATISTICAL ANALYSIS}

Continuous variables were expressed as mean \pm standard deviation, categorical data were expressed as numbers and percentages. Normality analyses were performed by Kolmogorov-Smirnov Goodness of Fit Test for intergroup analysis of continuous variables.

One Way Analysis of Variance (One-way ANOVA) was used for comparisons between three and more groups and T-test was used for comparisons between two groups. Pearson Correlation Coefficient was used to test the linear correlation between the variables. Chi-square test was used to compare categorical data. Analyses were performed with Statistical Package for Social Sciences version 15.0 (SPSS Inc., Chicago, IL, USA). Statistical significance level was considered as $p<0.05$.

\section{RESULTS}

The patients with a migraine attack, tension-type headache and the migraine patients in remission were asked about the character, localization, lateralization and accompanying symptoms of headache. The findings were recorded in the study question- 
naire. Patients presenting with tension-type headache described compressive headache, while $82.6 \%$ of all patients in the migraine episode and migraine attack group had throbbing headache in accordance with the migraine clinic. When the localization of the headache was questioned, the frontal localization was described in $72 \%$ of the tension headache group. $90 \%$ of tension headache group showed bilateral localization, 70\% right and left unilateral headache in migraine remission group and $65 \%$ right and left unilateral headache in migraine attack group. When the groups were examined in terms of concomitant symptoms, tension-type headache group had $40 \%$ complaints of nausea, and 35\% had photophobia and phonophobia. Nausea was detected in $88 \%$ of patients with migraine $(n=43)$; accompanied by headache. Nausea and photophobia were present in all migraine remission group. $78 \%$ of the migraine attack group had nausea and photophobia. Phonophobia was among the accompanying findings consistent with the migraine clinic in 36 patients (83.7\%) diagnosed with migraine. Paresis findings were present only in 1 patient in the remission migraine group.

While there was no statistically significant difference between the groups in terms of age and gender, BMI and family history in terms of the presence of statistically significant difference was detected. When the mean BMI of the participants in the migraine attack and tension-type headache groups was compared using One-way ANOVA test, it was found to be higher than the control group (Tab. 4).
When the right and left carotid intima-media thicknesses and mean CIMT values were obtained; mean right CIMT was $0.49 \pm 0.17$ in tension-type headache, $0.43 \pm 0.16$ in patients with migraine remission, $0.45 \pm 0.09$ in migraine attack group, and $0.49 \pm 0.14 \mathrm{~mm}$ in control group. The mean values of left CIMT were $0.44 \pm 0.15$ in GTBA, $0.43 \pm 0.17$ in migraine patients, $0.46 \pm 0.13$ in migraine attack group and $0.50 \pm 0.14 \mathrm{~mm}$ in control group, respectively. There was no statistically significant difference between the groups in the right and left CIMT measurements ( $p>0.05$ ) (Tab. 5).

There was no significant difference between the mean CIMT values $(p=0.489)$. The mean CIMT was calculated as $0.46 \pm 0.14$ in the GTBA, $0.43 \pm 0.15$ in the remission period, $0.45 \pm 0.10$ in the migraine attacks, and $0.50 \pm 0.12 \mathrm{~mm}$ in the control group. (Tab. 5)

Carotid intima-media thickness measurements according to migraine type were compared using T-test and no statistically significant difference was found ( $p>0.05$ ) (Tab. 6). The mean CIMT values of migraine patients with aura were $0.44 \pm 0.13 \mathrm{~mm}$ and $0.45 \pm 0.12 \mathrm{~mm}$ for migraine without aura $(p=0.701)$. (Tab. 6)

No statistically significant difference was found in the measurements of right and left CIMT using Mann Whitney test according to the presence of migraine ( $p=0.139, p=0.414$ ) (Tab. 7). The average carotid intima-media thickness measurement according to the presence of migraine by T-test was $0.48 \pm 0.13 \mathrm{~mm}$ in the absence of migraine and

\begin{tabular}{|c|c|c|c|c|c|}
\hline & Tension type & Migraine in remission & Migraine attack & Control & $p$ \\
\hline Age & $33.40 \pm 11.60$ & $33.00 \pm 11.13$ & $36.21 \pm 9.82$ & $31.95 \pm 9.34$ & $0.571^{*}$ \\
\hline Gender (F/M) & $11 / 9$ & $16 / 4$ & $16 / 7$ & $10 / 11$ & $0.133^{* *}$ \\
\hline BMI $\left(\mathrm{kg} / \mathrm{m}^{2}\right)$ & $26.85 \pm 4.93^{*}$ & $23.94 \pm 4.33$ & $26.44 \pm 4.74^{*}$ & $22.93 \pm 2.70^{*}$ & $0.009^{*}$ \\
\hline Family history & $20 / 0^{*}$ & $8 / 12^{*}$ & $15 / 8^{*}$ & $19 / 2$ & $<0.001^{* *}$ \\
\hline
\end{tabular}

\begin{tabular}{|c|c|c|c|c|c|}
\hline & TTH $(n=20)$ & $\operatorname{MR}(n=20)$ & $M A(n=23)$ & Control $(n=21)$ & $p$ \\
\hline Right CIMT (mm) & $0.49 \pm 0.17$ & $0.43 \pm 0.16$ & $0.45 \pm 0.09$ & $0.49 \pm 0.14$ & $0.446^{*}$ \\
\hline Left CIMT (mm) & $0.44 \pm 0.15$ & $0.43 \pm 0.17$ & $0.46 \pm 0.13$ & $0.50 \pm 0.14$ & $0.515^{*}$ \\
\hline Mean CIMT (mm) & $0.46 \pm 0.14$ & $0.43 \pm 0.15$ & $0.45 \pm 0.10$ & $0.50 \pm 0.12$ & $0.489 *$ \\
\hline
\end{tabular}




Table 6. Comparison of CIMT values by migraine-
type
\begin{tabular}{|l|l|l|c|}
\hline & $\begin{array}{l}\text { Migraine with } \\
\text { aura }(\mathrm{n}=22)\end{array}$ & $\begin{array}{l}\text { Migraine with } \\
\text { aura }(\mathrm{n}=21)\end{array}$ & $\mathrm{p}$ \\
\hline $\begin{array}{l}\text { Right CIMT } \\
(\mathrm{mm})\end{array}$ & $0.44 \pm 0.13$ & $0.44 \pm 0.12$ & $0.951^{*}$ \\
\hline Left CIMT $(\mathrm{mm})$ & $0.43 \pm 0.15$ & $0.46 \pm 0.15$ & $0.550^{*}$ \\
\hline $\begin{array}{l}\text { Mean CIMT } \\
(\mathrm{mm})\end{array}$ & $0.44 \pm 0.13$ & $0.45 \pm 0.12$ & $0.701^{*}$ \\
\hline
\end{tabular}

$0.44 \pm 0.13 \mathrm{~mm}$ in the presence of migraine (Tab. 7). There was no statistically significant difference between the groups in terms of the presence of migraine in the mean CIMT measurements $(p=0.202)$.

\section{DISCUSSION}

Carotid intima-media thickness measurement is a safe, inexpensive, non-invasive and easily applicable method, and recent studies have shown that there is a strong correlation between early changes secondary to atherosclerosis and carotid intima-media thickness [11, 12]. In a case-control study by Martinic-Popovic et al., a significant difference was found between carotid intima-media thickness between patients with transient ischaemic attack and control group, and CIMT measurements were higher in transient ischaemic attack group and it was defended that CIMT measurement could be used to determine the risk of cerebrovascular disease [13].

In the observational cohort study conducted by Beşir et al. in 2010 in Turkey, it was found that CIMT values in healthy volunteers increased only by age and it has been found that CIMT measurement can be used to evaluate early atherosclerosis burden in adult individuals [14]. Recent studies have shown that the risk of ischaemic stroke is high in patients with migraine, especially in women with migraine with aura $[15,16]$. Increased C-reactive protein (CRP), interleukins, tumour necrosis factor- $\alpha$ and adhesion molecules have been shown to be the underlying causes of oxidative stress, inflammation and thrombosis [17]. However, the relationship between migraine and cardiovascular and cerebrovascular disease is still not clear $[18,19]$.

In a study conducted by Tatar et al., carotid intima-media thickness was evaluated between 25 women with migraine and 27 healthy women with a mean age of $33 \pm 9$ years and In the migraine group, a statistically significant increase was found and the association of migraine patients with subclinical atherosclerosis was reported [10]. In a similar study by Hamed et al., between 44 migraine patients in remission, 19 tension-type headache patients and 35 control groups, the thickness of carotid intima-media were compared and CIMT measurements of migraine with and without aura were significantly higher than tension headache and control groups [20]. Again, in the same study, higher CIMT measurements were obtained in migraine without aura compared to those with aura [20]. Also, the participants had no cardiovascular disease or cardiovascular risk factor, as in our study. For example, in a multi-centre cohort study in Brazil, a total of 383 migraine patients with 163 aura and 220 without aura were included in the study and compared with healthy volunteers and subclinical atherosclerosis was investigated by looking at carotid intima-media thickness and coronary artery cal-

\begin{tabular}{|c|c|c|c|c|}
\hline & & $\begin{array}{l}\text { Right CIMT (mm) } \\
\qquad p=0.139\end{array}$ & $\begin{array}{l}\text { Left CIMT (mm) } \\
\qquad p=0.414\end{array}$ & $\begin{array}{c}\text { Mean CIMT K }(\mathrm{mm}) \\
\mathrm{p}=0.202\end{array}$ \\
\hline \multirow[t]{5}{*}{ Migraine $n=41$} & Mean & 0.49 & 0.47 & 0.48 \\
\hline & Median & 0.50 & 0.48 & 0.49 \\
\hline & Std deviation & 0.15 & 0.15 & 0.137 \\
\hline & Minimum & 0.19 & 0.22 & 0.20 \\
\hline & Maximum & 0.80 & 0.90 & 0.85 \\
\hline \multirow[t]{5}{*}{ No migraine $n=43$} & Mean & 0.44 & 0.45 & 0.44 \\
\hline & Median & 0.43 & 0.44 & 0.45 \\
\hline & Std deviation & 0.12 & 0.15 & 0.13 \\
\hline & Minimum & 0.19 & 0.19 & 0.19 \\
\hline & Maximum & 0.70 & 0.83 & 0.75 \\
\hline
\end{tabular}


cium score [21]. Cardiovascular disease risk factors (diabetes mellitus, hypertension, hyperlipidaemia, smoking/alcohol use, etc.) were not excluded in the study group, but the migraine group had no obvious cardiovascular disease in their medical history. In the same study, the mean age was 48 in the migraine group and according to the presence of migraine and the type of migraine, there was no statistical difference in terms of carotid intima-media and subclinical atherosclerosis was not associated with migraine.

In the cross-sectional case-control study conducted by Stam et al., the number of participants was higher and the control group with 360 migraines and 617 migraines was formed. To assess the risk of atherosclerosis in patients with migraine, CIMT, pulse wave velocity and ankle-brachial index (ABI), i.e. ankle / brachial systolic pressure ratio parameters were measured. These three parameters are non-invasive methods and are accepted as an indicator of atherosclerosis. A significant difference was not found in these three parameters in patients with migraine compared to the control group. At the end of the study, it was concluded that there is no increased risk of atherosclerosis in migraine patients [22].

In terms of age, exclusion criterion and number, there were no significant differences in CIMT in two smaller studies that were more consistent with our study $[23,24]$. One of these studies is a case-control study by Perko et al. The study included $40 \mathrm{mi}$ graine patients with 20 auras, 20 without aura and a control group of 20 people with a mean age of $35 \pm 8$ years. None of the study and control groups had comorbid characteristics as in the presented study. The patients with migraine were in the episode-free period. When compared between groups and migraine type, no significant difference was found in terms of CIMT [23].

In the study of Liman, there were no comorbid features in the resumes of the study group consisting of 30 healthy women and 29 women with migraine with aura. When evaluated in terms of CIMT and in addition peripheral arterial tonometry, it was concluded that there is no diagnostic value of CIMT in migraine and that migraine is not associated with subclinical atherosclerosis [24].

\section{CONCLUSION}

When we look at the pathophysiology of migraine, it is not wrong to say that there is a tendency to atherosclerosis due to chronic inflammation and increased thrombotic and vasoactive substances, but the results of the studies on this subject are quite different from each other, and many studies have shown that migraine disease is not associated with subclinical atherosclerosis.

In the authors' study, CIMT was investigated in all aspects of migraine and tension headache; migraine patients were compared in terms of both migraine type and presence of exacerbation. In this respect, our study is the first in the literature.

As a result of this study, migraine and tension headache patients do not have any relationship with subclinical atherosclerosis and CIMT measurement has no value in differential diagnosis. however, due to the controversial results that emerge when all the studies are considered, there is a need for further studies with larger working groups.

Funding sources: No funding was received.

Authors' contribution: Gülden Kazancı performed the study design, data collection, analysis, and article's drafting; Togay Evrin, Burak Katipoğlu, Refah Sayın performed study design, analysis and article's drafting; Ebru Ozan Sanhal performed study design, data collection and article's drafting; Ertuğrul Kazancı and Fatih Demir performed data collection and article's drafting, Semih Korkut and Lukasz Szarpak performed study design and article's drafting.

Conflict of interest: All authors declare that they have no conflict of interest.

Ethical approval: All procedures performed in studies involving human participants were in accordance with the ethical standards of the institutional and/or national research committee and with the 1964 Helsinki declaration and its later amendments or comparable ethical standards. This article does not contain any studies with animals performed by any of the authors.

\section{REFERENCES}

1. Yilmaz $M$, Gurger $M$, Atescelik $M$, et al. Meteorologic parameters and migraine headache: ED study. Am J Emerg Med. 2015; 33(3): 409-413, doi: 10.1016/j.ajem.2014.12.056, indexed in Pubmed: 25601162.

2. Zarifoglu MSA, Hayran OTG. An epidemiological study of headache in Turkey: a nationwide survey. Neurology. 1998; 50(A225).

3. Judith Tintinalli JS, 0. John Ma DC, Rita Cydulka GM. Tintinalli's Emergency Medicine: A Comprehensive Study Guide, Seventh Edition Emergency Medicine (Tintinalli). 7th edition. 
4. http://www.itfnoroloji.org/ekitap.htm.

5. Kanters SD, Algra A, van Leeuwen MS, et al. Reproducibility of in vivo carotid intima-media thickness measurements: a review. Stroke. 1997; 28(3): 665-671, doi: 10.1161/01.str.28.3.665, indexed in Pubmed: 9056629.

6. Glagov S, Weisenberg E, Zarins CK, et al. Compensatory enlargement of human atherosclerotic coronary arteries. N Engl J Med. 1987; 316(22): 1371-1375, doi: 10.1056/NEJM198705283162204, indexed in Pubmed: 3574413.

7. Touboul PJ, Elbaz A, Koller $C$, et al. Common carotid artery intima-media thickness and brain infarction : the Etude du Profil Génétique de I'Infarctus Cérébral (GENIC) case-control study. The GENIC Investigators. Circulation. 2000; 102(3): 313-318, doi: 10.1161/01. cir.102.3.313, indexed in Pubmed: 10899095.

8. O'Leary DH, Polak JF, Kronmal RA, et al. Carotid-artery intima and media thickness as a risk factor for myocardial infarction and stroke in older adults. Cardiovascular Health Study Collaborative Research Group. N Engl J Med. 1999; 340(1): 14-22, doi: 10.1056/ NEJM199901073400103, indexed in Pubmed: 9878640.

9. Parmeggiani L, Salandin $M$, Egger $F$, et al. A thicker intima-media carotid wall was found in a cohort of children with recent onset migraine. Acta Paediatr. 2016; 105(12): e577-e581, doi: 10.1111/ apa.13611, indexed in Pubmed: 27686570.

10. Güneş Tatar İ, Ergun 0 , Çeltikçi $P$, et al. Evaluation of subclinical atherosclerosis in migraine patients by ultrasound radiofrequency data technology: preliminary results. Agri. 2016; 28(3): 121-126, doi: 10.5505/agri.2016.00378, indexed in Pubmed: 27813037.

11. Simons PC, Algra A, Bots ML, et al. Common carotid intima-media thickness and arterial stiffness: indicators of cardiovascular risk in high-risk patients. The SMART Study (Second Manifestations of ARTerial disease). Circulation. 1999; 100(9): 951-957, doi: 10.1161/01. cir.100.9.951, indexed in Pubmed: 10468526.

12. Mackinnon $A D$, Jerrard-Dunne $P$, Sitzer $M$, et al. Rates and determinants of site-specific progression of carotid artery intima-media thickness: the carotid atherosclerosis progression study. Stroke. 2004; 35(9): 2150-2154, doi: 10.1161/01.STR.0000136720.21095.f3, indexed in Pubmed: 15243147.

13. Martinic-Popovic I, Simundic AM, Dukic L, et al. The association of inflammatory markers with cerebral vasoreactivity and carotid atherosclerosis in transient ischaemic attack. Clin Biochem. 2014; 47(1617): 182-186, doi: 10.1016/j.clinbiochem.2014.07.010, indexed in Pubmed: 25046654.
14. Beşir FH, Yazgan S, Celbek G, et al. [Normal value correlates of carotid intima- media thickness and affecting parameters in healthy adults]. Anadolu Kardiyol Derg. 2012; 12(5): 427-433, doi: 10.5152/ akd.2012.127, indexed in Pubmed: 22591938.

15. Schürks $M$, Rist PM, Bigal ME, et al. Migraine and cardiovascular disease: systematic review and meta-analysis. BMJ. 2009; 339: b3914, doi: 10.1136/bmj.b3914, indexed in Pubmed: 19861375.

16. Kurth $T$, Schürks $M$, Logroscino $G$, et al. Migraine, vascular risk, and cardiovascular events in women: prospective cohort study. BMJ. 2008; 337: a636, doi: 10.1136/bmj.a636, indexed in Pubmed: 18687721.

17. Sarchielli P, Alberti A, Baldi A, et al. Proinflammatory cytokines, adhesion molecules, and lymphocyte integrin expression in the internal jugular blood of migraine patients without aura assessed ictally. Headache. 2006; 46(2): 200-207, doi: 10.1111/j.15264610.2006.00337.x, indexed in Pubmed: 16492228.

18. Tietjen GE. Migraine as a systemic vasculopathy. Cephalalgia. 2009; 29(9): 987-996, doi: 10.1111/j.1468-2982.2009.01937.x, indexed in Pubmed: 19689607.

19. Vanmolkot FH, Van Bortel LM, de Hoon JN. Altered arterial function in migraine of recent onset. Neurology. 2007; 68(19): 1563-1570, doi: 10.1212/01.wnl.0000260964.28393.ed, indexed in Pubmed: 17460157.

20. Hamed SA, Hamed EA, Ezz Eldin AM, et al. Vascular risk factors, endothelial function, and carotid thickness in patients with migraine: relationship to atherosclerosis. J Stroke Cerebrovasc Dis. 2010; 19(2): 92-103, doi: 10.1016/j.jstrokecerebrovasdis.2009.04.007, indexed in Pubmed: 20189084.

21. Goulart AC, Santos IS, Bittencourt MS, et al. Migraine and subclinical atherosclerosis in the Brazilian Longitudinal Study of Adult Health (ELSA-Brasil). Cephalalgia. 2016; 36(9): 840-848, doi: 10.1177/0333102415613611, indexed in Pubmed: 26506847.

22. Stam $A H$, Weller $C M$, Janssens $A C$, et al. Migraine is not associated with enhanced atherosclerosis. Cephalalgia. 2013; 33(4): 228-235, doi: 10.1177/0333102412466966, indexed in Pubmed: 23147163.

23. Perko D, Pretnar-Oblak J, Sabovic M, et al. Endothelium-dependent vasodilatation in migraine patients. Cephalalgia. 2011; 31(6): 654-660, doi: 10.1177/0333102410390396, indexed in Pubmed: 21296807.

24. Liman TG, Neeb L, Rosinski J, et al. Peripheral endothelial function and arterial stiffness in women with migraine with aura: a case-control study. Cephalalgia. 2012; 32(6): 459-466, doi: 10.1177/0333102412444014, indexed in Pubmed: 22523187. 